\title{
New composites of betulin esters with arabinogalactan as highly potent anti-cancer agents
}

T.P. Shakhtshneider ${ }^{1,2}$, S.A. Kuznetsova ${ }^{3,4}$, A.S. Zamay ${ }^{3,5}$, T.N. Zamay ${ }^{5}$, E.A. Spivak ${ }^{5}$, M.A. Mikhailenko $^{1}$, Yu.N. Malyar ${ }^{3}$, B.N. Kuznetsov ${ }^{3,4}$, N.V. Chesnokov ${ }^{3,4}$, V.V. Boldyrev ${ }^{1,2}$

${ }^{1}$ Institute of Solid State Chemistry and Mechanochemistry SB RAS, Kutateladze 18, Novosibirsk, 630128 Russia;

${ }^{2}$ Novosibirsk State University, Pirogov str., 2, Novosibirsk, 630090 Russia;

${ }^{3}$ Institute of Chemistry and Chemical Technology SB RAS, Krasnoyarsk, Russia;

${ }^{4}$ Siberian Federal University, Krasnoyarsk, Russia;

${ }^{5}$ Research Institute of Molecular Medicine and Pathobiochemistry, Krasnoyarsk State Medical University, Krasnoyarsk, Russia.

\begin{abstract}
Betulin and its esters are the natural compounds with high in vitro cytotoxicity toward many cancer cells. However, the poor water solubility of these compounds has limited their applications. We prepared new composites of betulin esters using two methods, namely ball milling of the mixtures of betulin esters with arabinogalactan and preparation of thin films of these mixtures by evaporating the aqueous solutions. These composites revealed higher water solubility as compared to the initial substances without losing the structural integrity and functionality of these compounds. As a result, the new composites have shown much higher inhibitory effects against different cancer cell lines such as Ehrlich ascite carcinoma cells and lung carcinoma cells (A549) in comparison with the initial substances. The cell viability studies based on Annexin V and Propidium iodide probes have confirmed the high proapoptotic effect of betulin esters derivatives against cancer cells.
\end{abstract}

Keywords: betulin diacetate, betulin dipropionate, arabinogalactan, composites, solubility, anticancer activity

\section{Introduction}

Betulin and the related compounds found in the outer bark of the birch tree have attracted an increasing interest in the pharmaceutical formulation, due to their versatile biological activity (Yogeeswari \& Sriram 2005; Alakurtti et al. 2006). The poor solubility of betulin and its derivatives in water has limited their applications. Therefore new approaches are required to increase rate of dissolution and solubility of betulin and its derivatives. In some works 
(Hertrampf et al. 2012; Wang et al. 2012), the preparation of the guest-host type complexes of betulin and betulinic acid with cyclodextrins (CD) were suggested using $\beta$-, Hydroxypropyl- $\beta$ $\mathrm{CD}, \gamma-$ and HP- $\gamma-\mathrm{CD}$ as well as the hydrophilic $\gamma$-cyclodextrin thioethers as host molecules. In (Drag-Zalesinska et al. 2009), the structures of betulin and betulinic acid were modified by transformation to mono- and disubstituted esters of L-amino acids. This allowed better water solubility to be achieved without loss of the anticancer properties. It has been shown also (Son et al. 1998) that up to $10 \mathrm{~mol} \%$ of betulinic acid may be entrapped in liposomes. The presence of polyvinylpyrrolidone or Proxanol increased the resistance of betulinic acid-containing liposomes to aggregation. These polymers solubilized betulinic acid with the same efficiency as liposomes.

The mechanical activation of drugs with the polymers is a well-known effective method for preparing powder systems which are characterized by an increased rate of dissolution and solubility of the drugs (Colombo et al. 1998; Shakhtshneider \& Boldyrev 1999). In our previous studies (Mikhailenko et al. 2010; 2011), the mechanocomposites of betulin with water-soluble synthetic polymers, polyvinylpyrrolidone and polyethylene glycol, were prepared that showed higher solubility of betulin and its gastroprotective properties. The purpose of the present work was to prepare the composites of betulin diacetate (BDA) and betulin dipropionate (BDP) (Figure 1) with the water-soluble natural polymer arabinogalactan.

Arabinogalactan (AG) (Figure 2) is a polysaccharide that is isolated from larch wood and exhibits several unique properties (Paulsen \& Barsett 2005). Preparation of intermolecular AG complexes with poorly water-soluble drugs enabled their pharmacological properties to be improved (Dushkin et al. 2008; Tolstikova et al. 2009; Khvostov et al. 2013). In a previous work (Shakhtshneider et al. 2013), we obtained the composites of AG with BDA by ball-milling. It has been shown that mechanocomposites of $\mathrm{AG}$ with $\mathrm{BDA}$ were capable of inducing elimination processes of Ehrlich ascites carcinoma cells. Complexes of BDA with AG were also obtained as the thin films that were readily soluble in water.

The present work focuses on the preparation and characterization of composites of AG with BDA and BDP as the thin films and comparison of their pharmacological properties with the activity of mechanocomposites using in vitro tests.

\section{Results and Discussion}

Scanning electron microscopy (SEM) was used to assess the particle morphology and possibility of interaction between the components. In the SEM photographs, the pure drugs, BDA (Supplementary Figure S1(a)) and BDP (Supplementary Figure S1(c)), are characterized by the presence of crystalline particles of a regular size and parallelepipedic shape. The SEM pictures of mechanically activated mixtures (Supplementary Figures S3(b), (d)) show a characteristic 
morphology as the small-sized particles tended to aggregate with possible interaction at the contacts of the particles.

The powder X-ray diffraction patterns (Supplementary Figure S1) of the milled mixtures of BDA and BDP with AG showed that betulin esters phases were amorphous after milling, thus suggesting the distribution of BDA and BDP in the polymer with formation of the composites. This may be the distribution of molecules (or molecular ensembles) of betulin ester in the polymer as supposed for some other low molecular weight drug substances (Dushkin et al. 2008). The stability of this composite at heating (Drebushchak et al. 2014) is possibly due to complexation of the components under ball-milling.

In the IR spectra of the ball-milled BDA-AG mixtures, as well as of the composites as the films, it is seen that the profile of $\mathrm{C}=\mathrm{O}$ stretching vibration in the BDA molecule changed in comparison with the physical mixture of the initial compounds (Figure 3(a)). This fact suggested hydrogen bonds formation at least for the part of the molecules of BDA in the ball-milled mixtures and in the films. In the case of the ball-milled BDP - AG mixtures, the effect was more pronounced; new bands appeared at the lower frequencies (Figure 3(b)).

It was found that in the case of the mechanocomposites, the solubility of betulin derivatives increased in comparison with the initial substances. Upon dissolution of the ballmilled mixtures in water, the concentration of BDA increased up to 8 times (Shakhtshneider et al. 2013) whereas the concentration of BDP increased up to near 4 times. The dynamics of dissolution of BDP from the physical and ball-milled mixtures with AG is presented in Supplementary Figure S3 (online only). It is seen that in the case of ball-milled mixture, the rate of dissolution and concentration of BDP in solution was higher in comparison with those for the physical mixture.

Ball-milling AG resulted in decreasing the molecular weight of the polymer as was observed also for AG treated in other mills (Dushkin et al. 2008). The same effect was observed for the ball-milled mixtures of BDA with AG (Figure 4). Dissolution of ball-milled AG in water followed by evaporation of the solvent led to partial restoration of molecular weight. Gelpermeation chromatogram of the BDA - AG ball-milled mixture after dissolution in water and subsequent solvent evaporation acquired the shape of that for starting AG suggesting the crosslinking the polymer chains due to formation of intermolecular complexes in solution. The existence of complexes in solution probably is facilitated by conformational flexibility of the polymer chains and formation of coils containing at least two chains. Moreover, the treatment with ultrasound resulted in the absence of the effect of restoration of molecular weight due to breaking the coils. 
Samples obtained by evaporating aqueous solutions of AG and its mixtures with BDA (or BDP) were slightly colored (yellowish) amorphous films of thickness near $10 \mu \mathrm{m}$. These films were transparent and flexible. It is seen from SEM photographs (Supplementary Figure S4(a)) that the surface of the films was rather smooth, disordered internal structure being visible on the cut. ASM studies showed (Supplementary Figure S4(b), (c)) that the surface of the films consisted of homogeneous globules of spherical or oblong shape with transverse dimensions of $100-200 \mathrm{~nm}$.

The changes in the IR spectra (Figure 3(a)) of the film in comparison with the spectra of the physical mixture suggested complexation between the components. The films were quickly and totally soluble in water using much less solvent than was used for their preparation. The content of betulin diacyles in the films was equal to about $2-2.5 \%$ of the mass of the film producing water solutions with BDA (or BDP) concentrations of $0.15-0.18 \mathrm{mg} / \mathrm{mL}$.

The inhibitory effect of betulinic acid and its derivatives only on cancer cells has been already described in many studies (Chintharlapalli et al. 2011; Potze et al. 2014). Previously we showed antitumor activity of BDA-AG complexes that activated apoptosis in murine Ehrlich ascites tumor cells by increasing the concentration of intracellular calcium and sodium (Shakhtshneider et al. 2013). We proposed that better solubility of the mechanocomposites and the films of BDA and BDP with AG could result in greater effect on the cells.

In this study, we evaluated the ability of betulin composites to induce apoptosis and necrosis in cancerous and normal cell cultures. In order to understand the mechanisms of cell death, the content of active oxygen species (ROS) was assessed.

The effects of pure BDA and BDP, BDA (BDP) - AG mechanocomposites and BDA (BDP) - AG films were determined in vitro on human lung adenocarcinoma A549 cells and control normal lung epithelial MRC5 cells (Supplementary Tables S1, S2). Studies have shown that pure BDA (or BDP) and AG as well as their physical mixtures, mechanocomposites and the films in varying degrees caused translocation of phosphatidylserine in the cells of lung adenocarcinoma A549, indicating the beginning of the apoptotic process (Figure 5). Interestingly, the antitumor activity of pure BDP was significantly higher than that of pure BDA. For the physical mixtures, the amount of apoptotic cells was equal or higher than that for pure drugs. Nevertheless, taking into account that the content of the drugs in the mixtures was 10 times less, it is evident that the presence of $\mathrm{AG}$ facilitated the activity of betulin esters. Comparing the activities of the physical mixtures of BDA and BDP with AG with those of the mechanocomposites, it is seen that they were almost the same and even higher than for BDA-AG and BDP-AG mechanocomposites. For mechanocomposites and the physical mixtures, the formation of different molecular complexes can be proposed that are characterized by different 
stability and other physical-chemical properties. Moreover, BDA-AG and BDP-AG films induced apoptosis in $82 \%$ and $85 \%$ of cells in culture, respectively. This could be the result of increased dissolution rate, solubility and consequently better bioavailability of the composite films. It should be noted also that in contrast to mechanocomposites, in the case of the films, the substances were totally soluble. It is interesting that the films were more effective although the content of the active substance in them was in 4-5 time less than in the physical mixtures or mechanocomposites.

The cytotoxic effects of the new composites of betulin were evaluated on healthy epithelial lung cells (MRC5). All tested compounds did not show any influence on MRC5 cells which proved the targeted influence of betulin on cancer cells (Supplementary Tables S1, S2). This selective effect could be explained by the differences in reactive oxygen species (ROS) content in cancer and normal cells (Fulda \& Kroemer 2009). Betulin increases mitochondrial membrane permeability, and therefore the increased ROS level may lead to apoptosis. As we proposed, the initial level of ROS in the cancerous cells was significantly higher than in healthy ones and thus does not substantially depend on the influence of substances.

\section{Conclusions}

Using ball-milling, powder composites of betulin diacetate and betulin dipropionate with watersoluble polysaccharide arabinogalactan were obtained. They showed higher solubility in comparison with the initial substances. Possible reasons of increasing the solubility are disordering of crystal structures of biologically active substances and formation of molecular complexes with the polymer. The composites of betulin esters with arabinogalactan were also prepared by dissolution the mixtures of the components in water and subsequent solvent evaporation. The products obtained were amorphous thin films readily soluble in water.

In vitro studies revealed that mechanocomposites of betulin esters with arabinogalactan as well as the composites prepared as the water-soluble films exhibited higher antitumor activity in comparison with the initial substances. The composites of BDA and BDP with AG, especially in film form, showed targeted antitumor effect, causing apoptosis in cancer cells without affecting healthy cells. Apparently, the mechanism of BDA-AG and BDP-AG films action on cancer cells is similar to the betulinic acid and affect mitochondrial function increasing their permeability to reactive oxygen species.

\section{Acknowledgements}

This work was supported by the RFBR under Grant No. 14-03-31900-mol-a); the Russian Academy of Sciences under Grant No. 5.FSM-3; and the RF Ministry of Education and Sciences 
under Grant No. RFMEFI60714X0031). Authors are grateful to Dr. C.P. Jen for kindly providing A549 and MRC5 cell lines.

\section{References}

Alakurtti S, Mäkelä T, Koskimies S, Yli-Kauhaluoma J. 2006. Pharmacological properties of the ubiquitous natural product betulin. Europ. J. Pharm. Sci. 29:1-13.

Chintharlapalli S, Papineni S, Lei P, Pathi S, Safe S. 2011. Betulinic acid inhibits colon cancer cell and tumor growth and induces proteasome-dependent and -independent downregulation of specificity proteins (Sp) transcription factors. BMC Cancer. 11:371.

Colombo I, Grassi G, Grassi M. 1998. Drug mechanochemical activation. J. Pharm. Sci. 98:3961-3986.

Drag-Zalesinska M, Kulbacka J, Saczko J, Wysocka T, Zabel M, Surowiak P, Drag M. 2009. Esters of betulin and betulinic acid with amino acids have improved water solubility and are selectively cytotoxic toward cancer cells. Bioorg. Med. Chem. Lett. 19:4814-4817.

Drebushchak VA, Mikhailenko MA, Shakhtshneider TP, Drebushchak TN, Kuznetsova SA, Malyar YuN. 2014. Thermal properties of betulin dipropionate and its mixtures with polymers. J. Therm. Anal. Calorim. 115:2521-2525.

Dushkin AV, Meteleva ES, Tolstikova TG, Tolstikov GA, Polyakov NE, Neverova NA, Medvedeva EN, Babkin VA. 2008. Mechanochemical preparation and pharmacological activities of water-soluble intermolecular complexes of arabinogalactan with medicinal agents. Russ. Chem. Bull. 57:1299-1307.

Fulda S, Kroemer G. 2009. Targeting mitochondrial apoptosis by betulinic acid in human cancers. Drug Discovery Today 14:885-890.

Hertrampf A, Gruendemann C, Jaeger S, Laszczyk M, Giesemann T, Huber R. 2012. In vitro cytotoxicity of cyclodextrin-bonded birch bark extract. Planta Medica. 78:881-889.

Khvostov MV, Chernonosov AA, Tolstikova TG, Kasakin MF, Fedorova OS, Dushkin AV. 2013. Effect of complexation with arabinogalactan on pharmacokinetics of "guest" drugs in rats: For Example, Warfarin. Biomed Res Int. [Internet]. [cited 2013 Jul 5]; Dec 17. Available from: http://dx.doi.org/10.1155/2013/156381.

Mikhailenko MA, Shakhtshneider TP, Brezgunova ME, Kuznetsova SA, Boldyrev VV. 2010 Oct 10. The composition of betulin with biocompatible polymers and the method of its preparation. Russuian Federation patent RF 401118.

Mikhailenko MA, Shakhtshneider TP, Drebushchak VA, Kuznetsova SA, Skvortsova GP, Boldyrev VV. 2011. Influence of mechanical treatment on the properties of betulin, betulin diacetate, and their mixture with water-soluble polymers. Chem. Nat. Comp. 47:229-233. 
Paulsen BS, Barsett H. Editor Heinze T. 2005. Bioactive pectic polysaccharides. Polysaccharides 1: Structure, characterization and use. Book Series: Advances in Polymer Science. 186:69101.

Potze L, Mullauer FB, Colak S, Kessler JH, Medema JP. 2014. Betulinic acid-induced mitochondria-dependent cell death is counterbalanced by an autophagic salvage response. Cell Death and Disease. [Internet]. [cited 2014 Apr 5];Feb 26. Available from: http://www.nature.com/cddis/journal/v5/n4/full/cddis2014139a.html

Shakhtshneider TP, Boldyrev VV. 1999. Mechanochemical Synthesis and Mechanical Activation of Drugs. In: Boldyreva EV, Boldyrev VV. Reactivity of Molecular Solids. Chichester: John Wiley \& Sons; p. 271.

Shakhtshneider TP, Kuznetsova SA, Mikhailenko MA, Zamai AS, Malyar YuN, Zamai TN, Boldyrev V.V. 2013. Effect of mechanochemical treatment on physicochemical and antitumor properties of betulin diacetate mixtures with arabinogalactan. Chem. Nat. Comp. 49:470-474.

Son LB, Kaplun AP, Spilevskii AA, Andiia-Pravdivyi IE, Alekseeva SG, Gribor'ev VB, Shvets VI. 1998. Synthesis of betulinic acid from betulin and study of its solubilization using liposomes. Bioorganicheskaya khimiya. 24:787-793.

Tolstikova TG, Khvostov MV, Bryzgalov AO. 2009. The complexes of drugs with carbohydratecontaining plant metabolites as pharmacologically promising agents. Mini-Reviews in Med. Chem. 9:1317-1328.

Wang HM, Şoica CM, Wenz G. 2012. A comparison investigation on the solubilization of betulin and betulinic acid in cyclodextrin derivatives. Nat. Prod. Commun. 7:289-291.

Yogeeswari P, Sriram D. 2005. Betulinic acid and its derivatives: A review on their biological properties. Curr. Med. Chem. 12:657-666. 


\section{Captions to Figures}

Figure 1. Betulin diacetate $\left(\mathrm{R}=\mathrm{CH}_{3}\right)$ and betulin dipropionate $\left(\mathrm{R}=\mathrm{CH}_{2} \mathrm{CH}_{3}\right)$ molecular structure.

Figure 2. Arabinogalactan molecular structure.

Figure 3. ATR spectra: a) 1:9 BDA-AG mixture initial (1) and ball-milled for 15 min (2), BDAAG film obtained by evaporation of an aqueous solution (3); b) BDP ball-milled for 30 min, 1:9 BDP-AG mixture ball-milled for 15 (2) and 30 min (3).

Figure 4. Gel-permeation chromatograms of starting AG (1); ball-milled AG (2); ball-milled AG (3) and ball-milled 1:9 BDA - AG mixture after evaporation of an aqueous solution (4).

Figure 5. Apoptotic activity of the initial BDA and BDP (1), physical (2) and ball-milled (3) 1:9 BDA(BDP) - AG mixtures, BDA(BDP) - AG films obtained by evaporation of aqueous solutions (4) in comparison with the intact cells (5). 